\title{
A hand hygiene intervention to reduce infections in child daycare: a randomized controlled trial
}

\author{
T. P. ZOMER ${ }^{1,2}$, V. ERASMUS ${ }^{2}$, C. W. LOOMAN ${ }^{2}$, A. TJON-A-TSIEN ${ }^{1}$, \\ E. F. VAN BEECK ${ }^{2}$, J. M. DE GRAAF ${ }^{2}$, A. H. E. VAN BEECK ${ }^{2}$, \\ J. H. RICHARDUS ${ }^{1,2}$ AND H. A. C. M. VOETEN ${ }^{1,2}$ \\ ${ }^{1}$ Department of Infectious Disease Control, Municipal Public Health Service Rotterdam-Rijnmond, Rotterdam, \\ The Netherlands \\ ${ }^{2}$ Department of Public Health, Erasmus MC, University Medical Centre Rotterdam, Rotterdam, The \\ Netherlands
}

Received 8 August 2014; Final revision 31 October 2014; Accepted 13 November 2014; first published online 8 January 2015

\section{SUMMARY}

Infections are common in children attending daycare centres (DCCs). We evaluated the effect of a hand hygiene $(\mathrm{HH})$ intervention for caregivers on the incidence of gastrointestinal and respiratory infections in children. The intervention was evaluated in a two-arm cluster randomized controlled trial. Thirty-six DCCs received the intervention including HH products, training sessions, and posters/stickers. Thirty-five control DCCs continued usual practice. Incidence of episodes of diarrhoea and the common cold in children was monitored by parents during 6 months. Using multilevel Poisson regression, incidence rate ratios (IRRs) with 95\% confidence intervals (CIs) were obtained. Diarrhoeal incidence was monitored in 545 children for 91937 days. During follow-up, the incidence was 3.0 episodes per child-year in intervention DCCs vs. $3 \cdot 4$ in control DCCs (IRR $0 \cdot 90,95 \%$ CI $0 \cdot 73-1 \cdot 11$ ). Incidence of the common cold was monitored in 541 children for 91373 days. During follow-up, the incidence was $8 \cdot 2$ episodes per child-year in intervention DCCs vs. $7 \cdot 4$ in control DCCs (IRR 1.07, 95\% CI 0.97-1·19). In this study, no evidence for an effect of the intervention was demonstrated on the incidence of episodes of diarrhoea and the common cold.

Key words: Cold (common), gastroenteritis, hand hygiene, hygiene - professional, infectious disease control.

\section{INTRODUCTION}

Children attending daycare centres (DCCs) acquire gastrointestinal and respiratory infections more often than children cared for at home $[1,2]$. Hand hygiene $(\mathrm{HH})$ is considered to be a simple and effective measure

\footnotetext{
* Author for correspondence: T.P. Zomer, Department of Infectious Disease Control, Municipal Public Health Service Rotterdam-Rijnmond, P.O. Box 70032, 3000 LP Rotterdam, The Netherlands.

(Email: t.zomer@rotterdam.nl)
}

to prevent infections [3, 4]. However, in DCCs caregivers' compliance with $\mathrm{HH}$ guidelines is low [5].

Although several $\mathrm{HH}$ interventions have been developed to reduce DCC-related infections [6-14], inconsistent results on their effectiveness have been reported [15]. Moreover, these interventions were not reported as being developed according to a stepwise behavioural approach using models and theories from the behavioural sciences to understand the determinants that underlie HH behaviour [16]. Our previous research showed that environmental determinants, such

This is an Open Access article, distributed under the terms of the Creative Commons Attribution licence (http://creativecommons.org/licenses/by/3.0/), which permits unrestricted re-use, distribution, and reproduction in any medium, provided the original work is properly cited. 
as the availability of paper towels, are associated with caregivers' HH compliance in DCCs [5]. In addition, we found that the following sociocognitive determinants are associated with $\mathrm{HH}$ compliance of DCC caregivers: knowledge and awareness of $\mathrm{HH}$ guidelines, perceived importance of performing $\mathrm{HH}$, perceived behavioural control (i.e. perceived ease or difficulty of performing the behaviour), and habit [17]. Interventions aiming to improve caregivers' HH compliance in DCCs are more likely to be successful when addressing these determinants. We therefore developed an intervention based on these sociocognitive and environmental determinants of caregivers' $\mathrm{HH}$ behaviour. The aim of the intervention was to increase caregivers' compliance with $\mathrm{HH}$ guidelines (primary outcome measure) and reduce infections in children (secondary outcome measure). Due to the intervention, caregivers' compliance with $\mathrm{HH}$ guidelines improved. Compliance was defined as the number of $\mathrm{HH}$ actions divided by the total number of opportunities for which $\mathrm{HH}$ was indicated. According to the Dutch national guidelines, $\mathrm{HH}$ is mandatory for caregivers before touching/preparing food, before caregivers themselves ate or assisted children with eating, and before wound care; and after diapering, after toilet use/wiping buttocks, after caregivers themselves coughed/sneezed/wiped their own nose, after contact with body fluids (e.g. saliva, vomit, urine, blood, or mucus when wiping children's noses), after wound care, and after hands were visibly soiled [18]. HH compliance was observed at 1, 3 and 6 months follow-up. At 6 months follow-up, caregivers' HH compliance in intervention DCCs was 59\% vs. 44\% in control DCCs (baseline-corrected OR 4·13, 95\% CI 2·33-7·32) (T. P. Zomer et al., unpublished data). The effect of our intervention on $\mathrm{HH}$ compliance will be described in a forthcoming paper. In this paper we assess the effect of our intervention on incidence of gastrointestinal and respiratory infections in children attending DCCs.

\section{METHODS}

A cluster randomized controlled trial of a $\mathrm{HH}$ intervention was performed in DCCs in the regions of Rotterdam-Rijnmond, Gouda and Leiden in The Netherlands between September 2011 and April 2012. DCCs were randomized, stratified for size and urbanicity [19]. DCCs which participated in our previous study on $\mathrm{HH}$ determinants [5, 17], were contacted to participate in the trial.

The intervention consisted of four components [19]. First, the following $\mathrm{HH}$ products were provided free of charge: dispensers for paper towels, soap, alcoholbased hand sanitizer and hand cream, with refills for 6 months. Second, training about the Dutch national $\mathrm{HH}$ guidelines was given and a booklet outlining the content of the training was distributed. Third, two team training sessions were given aimed at goalsetting and formulating specific $\mathrm{HH}$ improvement activities. The team training sessions were based on similar HH training sessions developed for Dutch hospitals [20, 21]. Fourth, posters and stickers for both caregivers and children were provided as reminders and cues to action. Two groups in each DCC participated in the study. In intervention DCCs, these groups received the $\mathrm{HH}$ products. As caregivers rotated between groups, all caregivers received the training sessions. The intervention was implemented in four phases (HH products at the start, three training sessions with a 1-month interval). Intervention DCCs were compared to control DCCs which continued their usual practice.

The outcome measure was incidence of gastrointestinal and respiratory infections in children monitored by parents. Parents were enrolled in the trial between 1 August 2011 and 1 November 2011. Baseline measurement was collected between mid-September 2011 until 1 November 2011; starting when parents were enrolled and ending when the intervention started. Follow-up measurement was from 1 November 2011 until the end of March 2012. Children were recruited from two groups of the DCC, even if the DCC had more than two groups in total. In that case, in both intervention and control DCCs the researchers in collaboration with the managers of the DCCs randomly selected two groups. Parents were recruited from 142 (48\%) groups out of a total of 297 groups. Children were eligible to participate if they: attended the DCC at least 2 days a week; were aged between 6 months and $3 \cdot 5$ years at start of the trial; intended to attend the DCC throughout the study period; and if their parents consented, were Dutch speaking, and had access to email or regular post. Children were excluded if they had a chronic illness or medication that predisposed them to infection, a sibling taking part in the trial (i.e. one child per family could be included), or if they started attending the DCC after start of the trial.

Parents were asked to monitor disease incidence in their child using an infection calendar to mark the days their child had diarrhoea and/or a common cold. Diarrhoea was defined as at least two watery or unusually loose stools in $24 \mathrm{~h}$. The common cold 
was defined as a blocked or runny nose with at least one of the following symptoms: coughing, sneezing, fever, sore throat, or earache. Every 2 weeks, parents were contacted by email and regular post to enter the calendar page in an online version of the calendar or to send it in using a free-of-charge return envelope. Parents who did not respond were reminded after 1 week (email), 2 weeks (letter), and 3 weeks (telephone). Sample size calculation showed that to be able to detect $25 \%$ reduction in incidence of gastrointestinal infections of three per year and $15 \%$ reduction in incidence of respiratory infections of nine per year, we would need disease monitoring of 600 children for 6 months ( $80 \%$ power, two-sided alpha of 0.05) [19].

In order to interpret results we assessed exposure to the intervention. We observed whether the intervention dispensers and posters/stickers were in use at 6 months' follow-up. In addition, a survey was conducted among caregivers.

Data were analysed using SPSS version 19 (SPSS Inc., USA) and R version 2.12.2 (R Foundation, Austria). Analyses were performed according to the intention-to-treat principle, i.e. including all intervention DCCs irrespective of whether they used the HH products, posters/stickers or completed all training sessions. First, baseline characteristics were compared using $\chi^{2}$ test or Fisher's exact test for categorical variables and independent $t$ test for continuous variables. Second, the incidence of diarrhoea and the common cold was calculated during baseline and follow-up in intervention and control DCCs. Incidence was defined as the number of disease episodes per child-year. A new disease episode was defined after seven symptom-free days and in additional analyses after three symptom-free days [9]. Episodes of illness which started on the first day parents started monitoring disease incidence were excluded.

Multilevel Poisson regression analyses were performed to correct for clustering of the data within DCCs. Incidence risk ratios (IRRs) with 95\% confidence intervals (CIs) were obtained for the intervention effect, corrected for DCC group type (0-1, $2-3,0-4$ years), as this was the only possible confounder that was shown to be significantly different between intervention and control DCCs/children at baseline. Besides overall incidence, incidence was calculated stratified for children aged $0-1$ and $2-3$ years. Tests for overdispersion were performed, but no corrections were necessary.

Additional analyses were performed to correct for baseline measurement. For this we calculated the interaction between intervention status of the DCC (i.e. intervention vs. control) and follow-up measurement (i.e. baseline $v$ s. follow-up). This resulted in an IRR for the difference between baseline and follow-up measurement in intervention DCCs and an IRR for the difference between baseline and follow-up in control DCCs. Comparison of these two IRRs resulted in a baseline-corrected IRR.

Ethical approval was waived by the Medical Ethics Committee of the Erasmus University Medical Centre in Rotterdam (MEC-2011-256).

\section{RESULTS}

In the trial 71 DCCs participated. After randomization, there were 36 intervention DCCs and 35 control DCCs. Of 1981 parents invited to participate, 766 gave informed consent for their child (response rate $39 \%$ ) (Fig. 1). Of 766 children, 553 were eligible for inclusion. Of 553 children, five parents did not return any of the calendar pages with incidence data and three parents did not return any pages during followup, therefore 545 children were included in the analyses. For 19 of 545 children, baseline incidence data were missing.

Of the 545 children, $278(51 \%)$ were in 34 intervention DCCs and $267(49 \%)$ in 35 control DCCs. The median number of participating children per DCC was seven (range 1-18). Of the 545 parents, $94 \%$ returned $\geqslant 12$ of 14 calendar pages. Comparison of baseline characteristics demonstrated that the group type significantly differed between intervention and control DCCs (Table 1). This variable was therefore included in further analyses as a possible confounder. None of the other baseline characteristics was significantly different between intervention and control DCCs and children (Table 1).

\section{Intervention exposure}

All 36 intervention DDCs received the training on $\mathrm{HH}$ guidelines and all, but two, received at least one of the team training sessions. Another two intervention DCCs did not use any of the provided HH products. The response rate to the questionnaire on intervention exposure was $50 \%$ (274/546). Of 274 caregivers, $79 \%$ attended at least one of the training sessions. The information booklet on $\mathrm{HH}$ guidelines was received by $77 \%$ of caregivers. At 6 months follow-up, the dispensers for paper towels, soap, alcohol-based hand sanitizer, and hand cream were used in at least one of two 


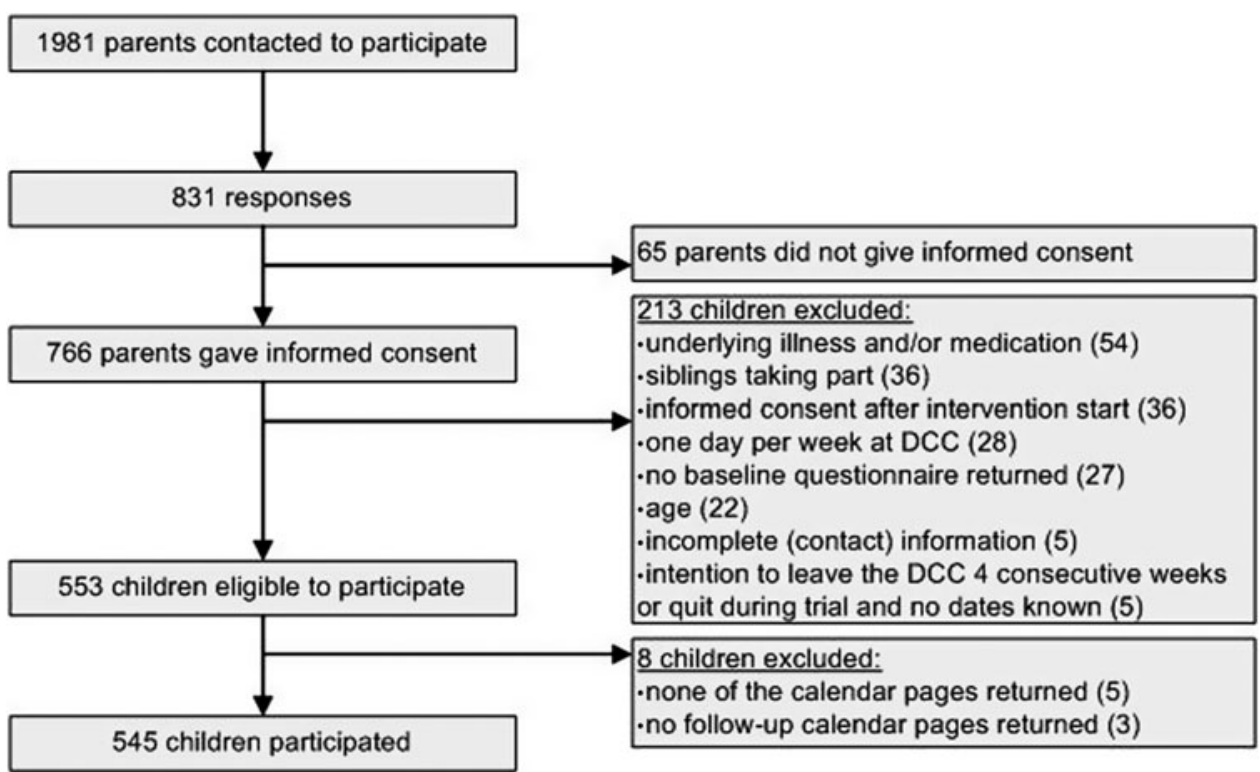

Fig. 1. Flow diagram of the recruitment of children in 71 child daycare centres (DCCs).

groups in respectively $94 \%, 89 \%, 86 \%$, and $45 \%$ of intervention DCCs. Moreover, in $86 \%$ the posters were used and in $74 \%$ the stickers.

\section{Incidence of episodes of diarrhoea and the common cold}

Incidence of episodes of diarrhoea was monitored in 545 children during 91937 days. Incidence of episodes of the common cold was monitored in 541 children during 91373 days. Of 545 children, four children were excluded from analyses because they had the common cold every day during the trial. Figure 2 shows the incidence of episodes of diarrhoea and the common cold in intervention and control DCCs over time; the crude incidence of diarrhoeal episodes differed between intervention and control DCCs at baseline, while during follow-up it was similar. Concerning the crude incidence of episodes of the common cold, at baseline as well as follow-up this was similar for intervention and control DCCs (except for November).

When defining a new episode of diarrhoea after seven symptom-free days, the incidence in intervention DCCs at baseline was 3.0 diarrhoeal episodes per child-year vs. $5 \cdot 1$ in control DCCs (Table 2). Corrected for group type and clustering of the data within DCCs, this difference was statistically significant (IRR 0.57, 95\% CI 0.38-0.85). During follow-up there were 3.0 diarrhoeal episodes per child-year in intervention DCCs vs. 3.4 in control DCCs (IRR 0.90, 95\% CI 0.73-1.11). The baseline-corrected IRR was $1 \cdot 58(95 \%$ CI 1·05-2.36). Additional analyses stratified by age, showed similar results during follow-up for children aged $0-1$ year (IRR $0 \cdot 97$, 95\% CI $0 \cdot 75$ 1.26; baseline-corrected IRR 1.82 , 95\% CI 1.08 3.08 ) and children aged 2-3 years old (IRR 0.83, 95\% CI 0.63-1.09; baseline-corrected IRR 1.29, 95\% CI 0.68-2.43) (results not shown in table). Analyses with a new disease episode defined after three symptom-free days, showed that uncorrected for baseline incidence there were slightly fewer episodes of diarrhoea in intervention vs. control DCCs (IRR 0.81, 95\% CI 0.63-1.05, $P=0 \cdot 07$ ) (Table 2).

When defining a new episode of the common cold after seven symptom-free days, the incidence in intervention DCCs at baseline was $9 \cdot 8$ episodes of the common cold per child-year vs. $9 \cdot 2$ in control DCCs (IRR $1 \cdot 06,95 \%$ CI $0 \cdot 85-1 \cdot 34$ ) (Table 3). During follow-up there were 8.2 episodes of the common cold in intervention DCCs vs. 7.4 in control DCCs (IRR 1.07, 95\% CI 0.97-1.19; baseline-corrected IRR 1.01, 95\% CI 0.79-1·29). Additional analyses stratified by age, showed similar results during follow-up for children aged $0-1$ year (IRR $1 \cdot 07,95 \%$ CI $0 \cdot 93-1 \cdot 22$; baselinecorrected IRR $1 \cdot 13,95 \%$ CI $0 \cdot 80-1 \cdot 61$ ) and children aged $2-3$ years (IRR $1 \cdot 10,95 \%$ CI $0 \cdot 95-1 \cdot 27$; baseline-corrected IRR $0.90,95 \%$ CI $0.63-1 \cdot 28$ ) (results not shown in table). Analyses with a new disease episode defined after three symptom-free days, showed similar results as analyses with a new disease episode after seven symptom-free days (IRR 1.04, 95\% CI 0.95-1·13; baseline-corrected IRR 1.05, 95\% CI 0.84-1·33) (Table 3). 
Table 1. Comparison of baseline characteristics of intervention and control daycare centres (DCCs) and children

\begin{tabular}{|c|c|c|c|}
\hline & Intervention DCCs & Control DCCs & $P$ value \\
\hline DCC characteristics & $(N=36)$ & $(N=35)$ & \\
\hline Size (large, having $\geqslant 46$ children per day) & $53 \%$ & $51 \%$ & $0 \cdot 91$ \\
\hline Degree of urbanicity & & & $0 \cdot 84$ \\
\hline Highly urban & $58 \%$ & $63 \%$ & \\
\hline Urban & $22 \%$ & $23 \%$ & \\
\hline Slightly/non-urban & $19 \%$ & $14 \%$ & \\
\hline Region & & & $0 \cdot 47$ \\
\hline Rotterdam-Rijnmond & $67 \%$ & $66 \%$ & \\
\hline Gouda & $14 \%$ & $6 \%$ & \\
\hline Leiden & $19 \%$ & $29 \%$ & \\
\hline Hygiene and quality certification (certified) & $44 \%$ & $41 \%$ & $0 \cdot 83$ \\
\hline Child characteristics & $\begin{array}{l}\text { Intervention children } \\
(N=278)\end{array}$ & $\begin{array}{l}\text { Control children } \\
(N=267)\end{array}$ & \\
\hline Gender (boys) & $51 \%$ & $54 \%$ & $0 \cdot 43$ \\
\hline Age at start of trial (mean) & 1.5 years & 1.6 years & $0 \cdot 53$ \\
\hline Children eating solid foods & $98 \%$ & $97 \%$ & $0 \cdot 74$ \\
\hline Children solely breastfed at start of trial & $0 \%$ & $2 \%$ & $0 \cdot 056$ \\
\hline Children ever breastfed & $76 \%$ & $79 \%$ & $0 \cdot 32$ \\
\hline Number of days per week at the DCC (mean) & $2 \cdot 7$ days & $2 \cdot 7$ days & $0 \cdot 70$ \\
\hline Children with siblings & $56 \%$ & $63 \%$ & $0 \cdot 09$ \\
\hline Children with siblings at the DCC & $25 \%$ & $26 \%$ & $0 \cdot 65$ \\
\hline $\begin{array}{l}\text { Children that started attending the DCC in the } \\
3 \text { months before trial start }\end{array}$ & $3 \%$ & $3 \%$ & $0 \cdot 96$ \\
\hline Children in a single-parent household & $7 \%$ & $9 \%$ & $0 \cdot 43$ \\
\hline DCC group type & & & $<0 \cdot 001$ \\
\hline $0-1$ years & $16 \%$ & $18 \%$ & \\
\hline $2-3$ years & $14 \%$ & $32 \%$ & \\
\hline $0-4$ years & $70 \%$ & $50 \%$ & \\
\hline
\end{tabular}

\section{DISCUSSION}

This is the first HH intervention in DCCs developed according to a stepwise behavioural approach targeting the underlying determinants of caregivers' compliance with $\mathrm{HH}$ guidelines. The study objective was to evaluate the effect of the intervention on the incidence of episodes of diarrhoea and the common cold in children attending DCCs. During follow-up, there were fewer episodes of diarrhoea in intervention DCCs vs. control DCCs. However, this difference was not statistically significant and corrected for baseline the effect changed direction, with significantly more diarrhoeal episodes in intervention DCCs. This was primarily influenced by an unexplainable high baseline incidence in control DCCs. No effect of the intervention was shown on the incidence of episodes of the common cold.

Our study has several strengths. This is the first $\mathrm{HH}$ intervention in DCCs which was developed based on the underlying determinants of $\mathrm{HH}$ behaviour. In addition, this is one of few DCC intervention studies correcting for baseline incidence in multilevel analyses. Other strengths of the study are the randomized controlled design, a large sample size of 71 DCCs, high exposure to intervention components, and a high percentage of calendar pages returned by parents with few children lost to follow-up. In addition, control DCCs received the intervention after data collection, which probably facilitated DCC recruitment and also minimized loss to follow-up [7].

Our study has several limitations. First, as the response of parents was initially limited, there were not enough children included when starting baseline measurement. Therefore, recruitment of parents continued during baseline measurement. As a result, the number of days that parents completed the infection calendar during baseline varies. For four children, no baseline data were provided by the parents. Moreover, the exact weeks that baseline data were collected vary between the children, which might lead to incomparable results due to different circulating pathogens. An additional complicating factor is that 


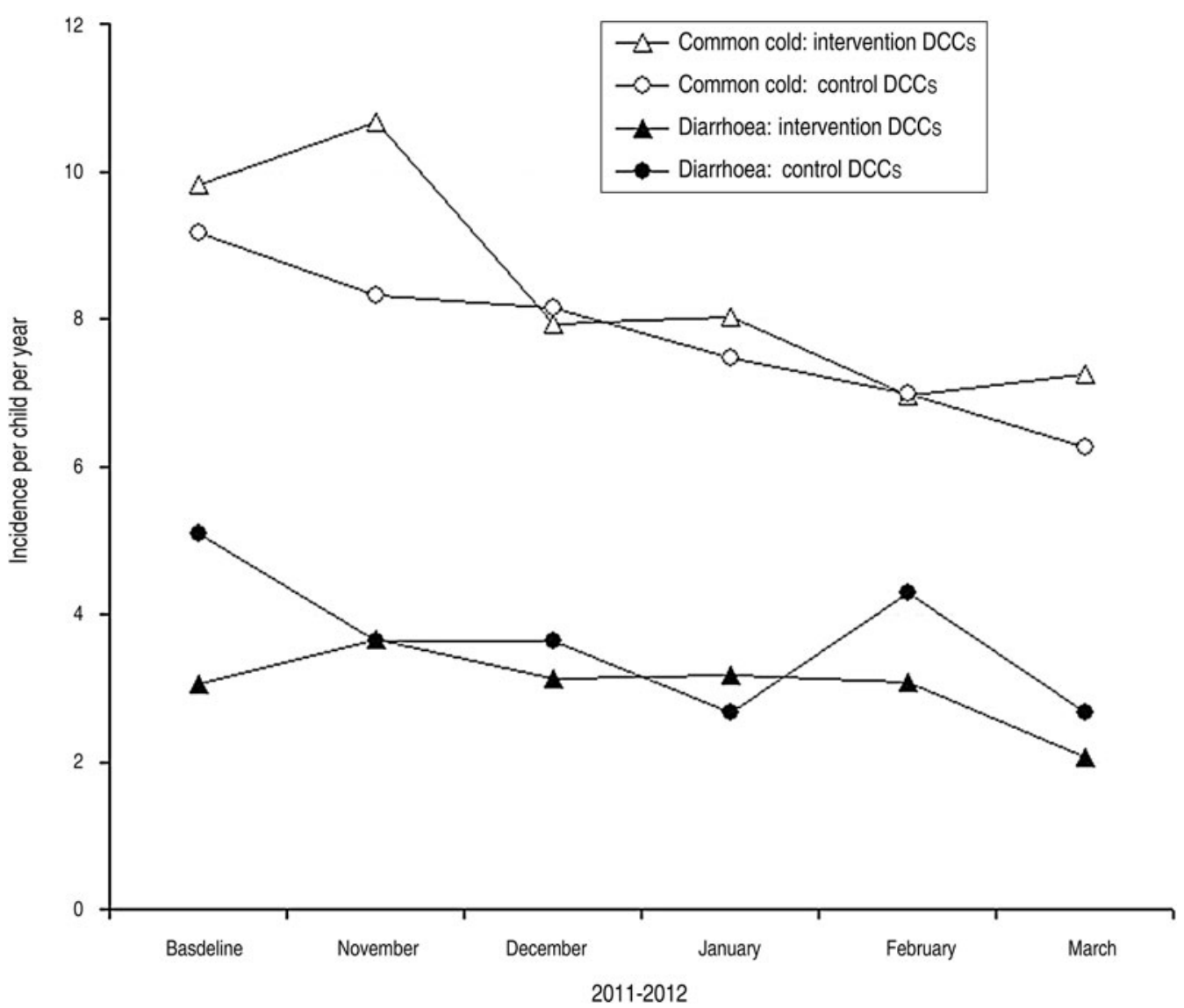

Fig. 2. Effect of a hand hygiene intervention on incidence of episodes of diarrhoea and the common cold in children attending daycare centres (DCCs).

half of intervention DCCs, due to practical reasons, had already received the training on $\mathrm{HH}$ guidelines while baseline measurement was still ongoing. For children of these DCCs, we censored the calendar days from the day of the training until the official intervention start on 1 November 2011. As a result, more baseline data were collected in control DCCs $v s$. intervention DCCs. The above-mentioned limitations of the baseline measurement might partly explain the baseline difference between intervention and control DCCs in incidence of episodes of diarrhoea. Another limitation is that our study is underpowered. According to sample size calculations, we would have needed disease monitoring of 600 children for 6 months (109 200 child-days) [19]. We monitored 545 children during 5 months follow-up, resulting in data on 79725 child-days, which is $73 \%$ of the anticipated 109200 child-days. Furthermore, the possible effect size of the intervention is probably smaller than what we assumed during sample size calculation. Other limitations are that the method to assess disease incidence was not validated, and the relatively low response rate in parents of $39 \%$. No information was obtained on parents unwilling to participate. It might be that these parents have less interest in hygiene which could have influenced disease incidence at child DCCs and possibly also the intervention effect.

In the same trial we also assessed caregivers' $\mathrm{HH}$ compliance at baseline and follow-up, and found that $\mathrm{HH}$ compliance increased significantly in intervention vs. control DCCs (T. P. Zomer et al., unpublished data). At baseline, compliance in intervention DCCs was $53 \%$ vs. $63 \%$ in control DCCs (OR 0.59, 95\% CI $0 \cdot 37-0 \cdot 94)$. At 6 months follow-up, compliance was $59 \%$ vs. $44 \%$, respectively (baselinecorrected OR $4 \cdot 13,95 \%$ CI $2 \cdot 33-7 \cdot 32$ ). Nevertheless, we were unable to demonstrate an effect of the intervention on incidence of episodes of diarrhoea and the common cold. This might partly be explained by the fact that on average the children attended the DCCs 2.7 days a week and children can also become infected outside the DCC. In The Netherlands it is common that at least one of the parents works parttime and therefore it is not surprising that the children 
Table 2. Effect of a hand hygiene intervention on incidence of episodes of diarrhoea in children attending daycare centres $(D D C s)(N=545)$

\begin{tabular}{|c|c|c|c|c|c|c|c|}
\hline & \multicolumn{2}{|c|}{ Intervention DCCs } & \multicolumn{2}{|c|}{ Control DCCs } & \multicolumn{2}{|c|}{ IRR* $(95 \%$ CI $)$} & \multirow[t]{2}{*}{$P$ value } \\
\hline \multicolumn{7}{|c|}{7 symptom-free days between episodes } & \\
\hline Baseline incidence $\dagger$ & $3 \cdot 0$ & $(42 / 5042)$ & $5 \cdot 1$ & $(100 / 7$ 170) & 0.57 & $(0 \cdot 38-0 \cdot 85)$ & $0 \cdot 006$ \\
\hline Follow-up incidence $\dagger$ & $3 \cdot 0$ & $(336 / 40564)$ & $3 \cdot 4$ & $(361 / 39161)$ & $0 \cdot 90$ & $(0 \cdot 73-1 \cdot 11)$ & $0 \cdot 32$ \\
\hline IRR* $(95 \% \mathrm{CI})$ & $1 \cdot 06$ & $(0 \cdot 76-1 \cdot 48)$ & $0 \cdot 67$ & $(0 \cdot 54-0 \cdot 84)$ & $1 \cdot 58$ & $(1 \cdot 05-2 \cdot 36)$ & 0.03 \\
\hline \multicolumn{8}{|c|}{3 symptom-free days between episodes } \\
\hline Baseline incidence $\dagger$ & $3 \cdot 2$ & $(44 / 5042)$ & $5 \cdot 7$ & $(112 / 7170)$ & 0.53 & $(0 \cdot 34-0 \cdot 83)$ & $0 \cdot 002$ \\
\hline Follow-up incidence $\dagger$ & $3 \cdot 3$ & (370/40 564) & $4 \cdot 1$ & $(435 / 39161)$ & $0 \cdot 81$ & $(0 \cdot 63-1 \cdot 05)$ & $0 \cdot 07$ \\
\hline IRR* $(95 \% \mathrm{CI})$ & $1 \cdot 11$ & $(0 \cdot 77-1 \cdot 60)$ & 0.72 & $(0 \cdot 57-0 \cdot 92)$ & $1 \cdot 53$ & $(1 \cdot 00-2 \cdot 36)$ & $0 \cdot 03$ \\
\hline
\end{tabular}

IRR, Incidence risk ratio; CI, confidence interval.

* Also corrected for clustering of the data within DCCs and group type.

$\dagger$ Incidence of episodes per year (i.e. no. of episodes/no. of days at risk).

Table 3. Effect of a hand hygiene intervention on incidence of episodes of the common cold in children attending daycare centres $(D D C s)(N=541)$

\begin{tabular}{|c|c|c|c|c|c|c|c|}
\hline & \multicolumn{2}{|c|}{ Intervention DCCs } & \multicolumn{2}{|c|}{ Control DCCs } & \multicolumn{2}{|c|}{ IRR* $(95 \%$ CI $)$} & \multirow{2}{*}{$P$ value } \\
\hline 7 symptom-free days bet & & & & & & & \\
\hline Baseline incidence $\dagger$ & $9 \cdot 8$ & $(132 / 4914)$ & $9 \cdot 2$ & $(178 / 7096)$ & $1 \cdot 06$ & $(0 \cdot 85-1 \cdot 34)$ & $0 \cdot 60$ \\
\hline Follow-up incidence $\dagger$ & $8 \cdot 2$ & (904/40 354) & $7 \cdot 4$ & $(794 / 39009)$ & $1 \cdot 07$ & $(0 \cdot 97-1 \cdot 19)$ & $0 \cdot 15$ \\
\hline IRR* $(95 \% \mathrm{CI})$ & $0 \cdot 83$ & $(0 \cdot 69-1 \cdot 00)$ & $0 \cdot 82$ & $(0 \cdot 70-0 \cdot 97)$ & $1 \cdot 01$ & $(0 \cdot 79-1 \cdot 29)$ & $0 \cdot 94$ \\
\hline \multicolumn{8}{|c|}{3 symptom-free days between episodes } \\
\hline Baseline incidence $\dagger$ & $11 \cdot 1$ & $(194 / 4914)$ & $11 \cdot 1$ & $(216 / 7096)$ & $0 \cdot 98$ & $(0 \cdot 80-1 \cdot 21)$ & $0 \cdot 87$ \\
\hline Follow-up incidence $\dagger$ & $9 \cdot 5$ & (1048/40 354) & 8.9 & $(955 / 39009)$ & $1 \cdot 04$ & $(0 \cdot 95-1 \cdot 13)$ & $0 \cdot 44$ \\
\hline IRR* $(95 \% \mathrm{CI})$ & $0 \cdot 86$ & $(0 \cdot 72-1 \cdot 02)$ & $0 \cdot 81$ & $(0.70-0.94)$ & $1 \cdot 05$ & $(0 \cdot 84-1 \cdot 33)$ & 0.65 \\
\hline
\end{tabular}

IRR, Incidence risk ratio; CI, confidence interval.

* Also corrected for clustering of the data within DCCs and group type.

$\dagger$ Incidence of episodes per year (i.e. no. of episodes/no. of days at risk).

only attend the DCC part-time. Another explanation could be that within DCCs other hygiene activities are also important for the prevention of disease transmission (i.e. cleaning toys, floors, furniture, toilets, etc.). Furthermore, in our study the main focus was on caregivers' $\mathrm{HH}$; besides posters and stickers for children, there were no other techniques to encourage children's HH, even though their HH might also be important to reduce infections in DCCs. Another possible explanation is that $\mathrm{HH}$ compliance did not improve enough in intervention DCCs to result in a reduction in infections, or that the difference in $\mathrm{HH}$ compliance between intervention and control DCCs was not large enough to detect differences in disease incidence. There is possibly a critical threshold for $\mathrm{HH}$ compliance to result in a lower incidence of infections. A Dutch study on DCC-related disease burden, during the same time period as our intervention showed a peak in incidence of gastroenteritis in February 2012 [22]. In our control DCCs there was the same increase in incidence, while this was not the case in intervention DCCs. Therefore, it might be that with an increase of infections, the intervention becomes more effective.

Our study shows the importance of baseline measurements in intervention studies, as baseline incidence of diarrhoeal episodes differed between intervention and control DCCs. There are few other DCC intervention studies which performed a baseline measurement $[6-8,13]$. One of these studies performed analyses to assess whether the difference between baseline and follow-up was different for intervention vs. control DCCs [8]. As we found a significant difference between intervention and control DCCs in diarrhoeal baseline incidence, we corrected the effect of the intervention for baseline incidence. 
This was done by adding an interaction term to assess whether the difference between baseline and follow-up was different for intervention vs. control DCCs. By adding this interaction term, we were still able to also correct for group type and clustering of the data within DCCs. To our knowledge, this is the first study to perform this type of analysis. More DCC intervention studies are needed with baseline measurement.

Previous HH intervention studies in DCCs have shown varying effects on incidence of gastrointestinal and/or respiratory infections and/or illness absenteeism [15]. We found three randomized controlled trials with an outcome measure of incidence of gastrointestinal and/or respiratory infections that corrected for clustering of the data $[6,9,10,13]$. Gudnason et al. also reported a baseline measurement and, similar to our study, did not demonstrate an effect of their intervention on incidence of diarrhoea and colds [6]. Roberts and colleagues reported a reduction in episodes of colds only in children aged $\leqslant 24$ months and a reduction in episodes of diarrhoea only in children aged $>24$ months $[9,10]$. However, in that study no baseline incidence was reported. Therefore, it is possible that the difference between intervention and control DCCs was already present before start of the intervention. Carabin et al. report that their intervention reduced the incidence of upper respiratory tract infections [13]. However, similar to our study, they also report a reduction in incidence in control DCCs. Therefore, it is less likely that the incidence reduction in intervention DCCs is caused by the intervention. This indicates that there is limited evidence available that improved $\mathrm{HH}$ in DCCs is associated with fewer gastrointestinal and respiratory infections. More evidence is needed to understand the importance of $\mathrm{HH}$ in reducing gastrointestinal and respiratory infections in children attending DCCs.

In conclusion, this study shows that there is no evidence that our HH intervention - addressing determinants that underlie caregivers' $\mathrm{HH}$ behaviour-is effective in reducing gastrointestinal and respiratory infections in children attending DCCs. An explanation might be that $\mathrm{HH}$ compliance did not increase enough to result in fewer infections and/or that other transmission routes are also important, such as other hygiene/cleaning activities within the DCC as well as children's HH. Future intervention studies should target several transmission routes and be evaluated in robust studies including baseline measurement.

\section{ACKNOWLEDGEMENTS}

We thank the DCCs and parents for their willingness to participate in the study. The study was by funded by the Netherlands Organization for Health Research and Development (ZonMw), project number 125020006. Dispensers and refills were sponsored by SCA Hygiene Products, Sweden.

[Trial registration: Dutch trials registry NTR3000.]

\section{DECLARATION OF INTEREST}

None.

\section{REFERENCES}

1. Zutavern A, et al. Day care in relation to respiratorytract and gastrointestinal infections in a German birth cohort study. Acta Paediatrica 2007; 96: 1494-1499.

2. Barros AJ. Child-care attendance and common morbidity: evidence of association in the literature and questions of design. Revista de Saude Publica 1999; 33: 98-106.

3. Ejemot RI, et al. Hand washing for preventing diarrhoea. Cochrane Database of Systematic Reviews 2008. Art. no. CD004265. Issue no. 1.

4. Jefferson T, et al. Physical interventions to interrupt or reduce the spread of respiratory viruses. Cochrane Database of Systematic Reviews 2011. Art. no.: CD006207. Issue no. 7.

5. Zomer TP, et al. Hand hygiene compliance and environmental determinants in child day care centers: an observational study. American Journal of Infection Control 2013; 41: 497-502.

6. Gudnason T, et al. Does hygiene intervention at day care centres reduce infectious illnesses in children? An intervention cohort study. Scandinavian Journal of Infectious Diseases 2013; 45: 397-403.

7. Rosen L, et al. Can a handwashing intervention make a difference? Results from a randomized controlled trial in Jerusalem preschools. Preventive Medicine 2006; 42: 27-32.

8. Ponka A, Poussa T, Laosmaa M. The effect of enhanced hygiene practices on absences due to infectious diseases among children in day care centers in Helsinki. Infection 2004; 32: 2-7.

9. Roberts L, et al. Effect of infection control measures on the frequency of diarrheal episodes in child care: a randomized, controlled trial. Pediatrics 2000; 105: 743-746.

10. Roberts $\mathbf{L}$, et al. Effect of infection control measures on the frequency of upper respiratory infection in child care: a randomized, controlled trial. Pediatrics 2000; 105: 738-742.

11. Kotch JB, et al. Evaluation of an hygienic intervention in child day-care centers. Pediatrics 1994; 94: 991-994.

12. Uhari M, Mottonen M. An open randomized controlled trial of infection prevention in child day-care centers. Pediatric Infectious Disease Journal 1999; 18: 672-677. 
13. Carabin $\mathbf{H}$, et al. Effectiveness of a training program in reducing infections in toddlers attending day care centers. Epidemiology 1999; 10: 219-227.

14. Hedin K, et al. Infection prevention at day-care centres: feasibility and possible effects of intervention. Scandinavian Journal of Primary Health Care 2006; 24: $44-49$.

15. Huskins WC. Transmission and control of infections in out-of-home child care. Pediatric Infectious Disease Journal 2000; 19: S106-110.

16. Bartholomew LK, et al. Planning Health Promotion Programs; An Intervention Mapping Approach. San Francisco: Jossey-Bass, 2011.

17. Zomer TP, et al. Sociocognitive determinants of observed and self-reported compliance to hand hygiene guidelines in child day care centers. American Journal of Infection Control 2013; 41: 862-867.

18. Dutch National Institute for Public Health and the Environment (RIVM), Dutch National Centre for
Hygiene and Safety (LCHV). Health risks in a child daycare centre or preschool (0 to 4 year olds) [in Dutch]. Bilthoven, The Netherlands. July 2011.

19. Zomer TP, et al. A hand hygiene intervention to decrease infections among children attending day care centers: design of a cluster randomized controlled trial. BMC Infectious Diseases 2013; 13: 259.

20. Huis A, et al. Impact of a team and leaders-directed strategy to improve nurses' adherence to hand hygiene guidelines: a cluster randomised trial. International Journal of Nursing Studies 2013; 50: 464-474.

21. Erasmus V, et al. The ACC OMPLISH study. A cluster randomised trial on the cost-effectiveness of a multicomponent intervention to improve hand hygiene compliance and reduce healthcare associated infections. BMC Public Health 2011; 11: 721.

22. Enserink R, et al. Infectious disease burden related to child day care in The Netherlands. Pediatric Infectious Disease Journal 2013; 32: e334-340. 